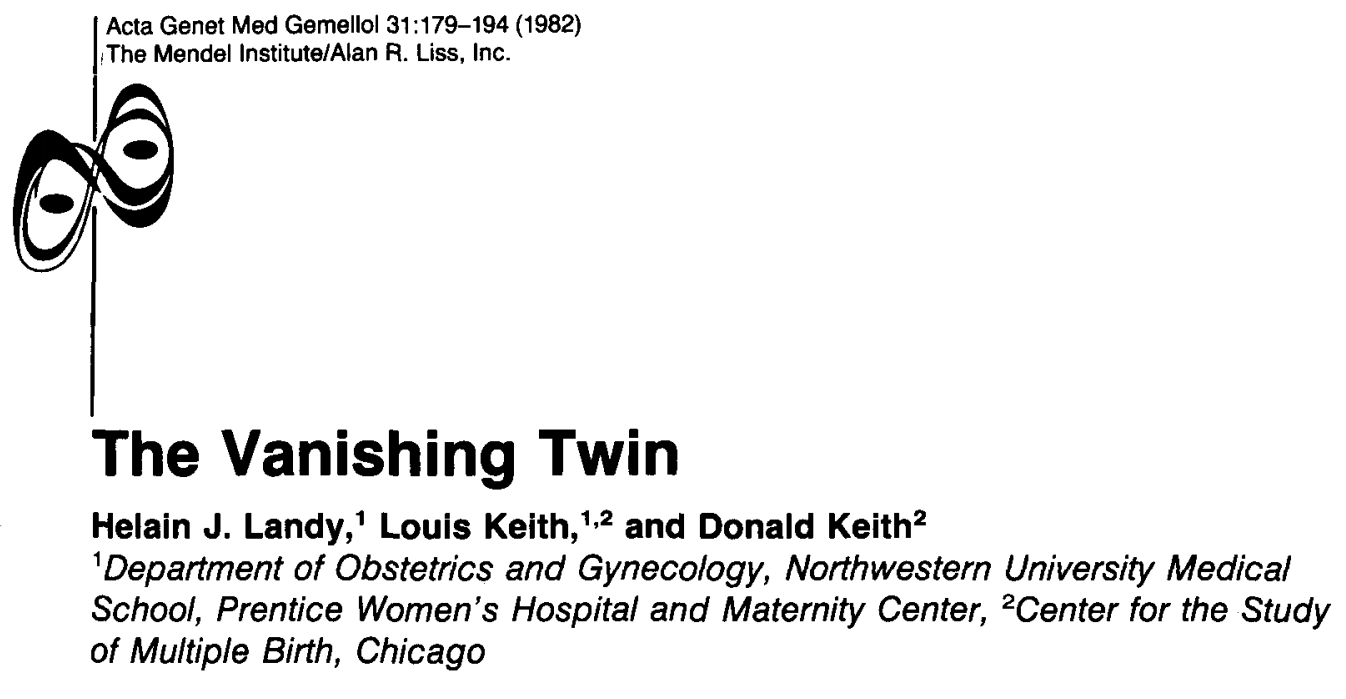

In general, it has not been recognized that many twin or multiple gestations are lost in utero early in pregnancy. Until the advent of ultrasound, the ability to document early human fetal loss in multiple gestation was difficult. However, recent reports of serial ultrasound examinations of pregnant women have documented the "disappearance" of at least one of two gestational rings. Furthermore, the number of twins observed at delivery was significantly less than the number of twin conceptions originally identified by ultrasound during the first trimester. These observations led to the concept of the "vanishing twin" $[8,10,13,15,17,20,22,35,41]$.

In order to obtain reference and personal data on this subject, we reviewed the literature and corresponded with members of the International Society for Twin Studies and obstetricians affiliated with Northwestern University Medical School, Chicago, Illinois. The nine studies that have documented this phenomenon cite "disappearance" rates ranging from $0 \%$ to $78 \%$, depending on patient population and timing of ultrasonography $[8,10,13,15,17,20,22,35,41]$. Several explanations are offered: physiological mechanisms of "disappearance" (resorption or formation of a blighted ovum or fetus papyraceus), artifactual error, incomplete scanning technique, and poor quality ultrasound equipment. The only complication thus far associated with "disappearance" of a fetus is slight vaginal bleeding.

Key words: Multiple gestation, Twins, First trimester ultrasonography, "Vanishing twin(s)", Blighted ovum, Resorption, Fetus papyraceus, First trimester bleeding

\title{
INTRODUCTION
}

Among human beings, the true conceptual rate of multiple gestation is difficult to determine with accuracy because of problems inherent in assessing: (1) the true rate of postconception pregnancy loss and (2) the frequency of twinning among pregnancies which result in early spontaneous abortions. Even though spontaneous abortions after six gestational weeks generally come to clinical attention, it has been suggested that from $43 \%$ to $78 \%$ of conceptions are lost at an earlier stage of gestation [27,31]. In fact, the prenatal elimination of very early gestations has been proposed as the main and most important method by which fetal or embryological abnormalities are discarded whether the pregnancy contains one or more gestational sacs.

This study was supported by the Center for the Study of Multiple Birth , Chicago, Illinois.

(C) 1982 Alan R. Liss, Inc. 
Several investigators have studied the frequency of twinning among spontaneously aborted gestational material; their results range from $0.3 \%$ to $20 \%[3,12,23]$. One recent study found 53 twin pairs among 1,939 spontaneous abortions, for a frequency of $2.7 \%$ [23].

Although it has been recognized that perinatal mortality is higher among multiple than among singleton pregnancies $[11,14,28]$ and that prematurity is the most common cause of death among twin infants born alive, it generally is not recognized that many twin or multiple pregnancies are lost in utero long before labor ensues [14,28]. More than a decade ago, Hewitt and Stewart suggested that the abortion risks for one member of a twin pregnancy are greater than the risks for both members and that the surviving twin is often mistaken for a singleton [11]. In those authors' opinion, misinterpreting the surviving twin as a singleton incorrectly skews data regarding both twinning frequencies and spontaneous abortion statistics [11].

The ability to document early human fetal loss in multiple gestation was problematic until the development of ultrasound. The diagnosis of multiple pregnancy has been made as early as the fifth week of gestation $[19,21]$, although reports of this finding are more common by the seventh week $[6,19]$. Gestational sacs can be visualized as ring echoes between approximately the fifth and the 12th weeks [30], crown-rump lengths can be assessed prior to the 13th week, and fetal heads can be seen after the 12th week of gestation [37].

Numerous reports of serial ultrasonographic examinations of pregnant women have demonstrated the "disappearance" of at least one of two gestational rings $[8,10,13,15$, $17,20,22,35,411$. Furthermore, when these women were followed to term, the number of twins observed at delivery was considerably less than the number of twin conceptions originally identified during the first trimester $[8,10,13,15,17,20,22,35,41]$. These observations have led to the concept of the "vanishing twin," a term which has gained a modest degree of acceptance in recent years and has provided biologists and clinicians with new information regarding the true incidence of human twinning. Because these data are scattered throughout diverse areas of medicine, a decision was made to review this literature. In addition, members of the International Society for Twin Studies were contacted for reference or personal data on this subject, and all obstetricians affiliated with Northwestern University Medical School in Chicago, Illinois, were contacted for data from their private cases.

\section{REVIEW OF THE LITERATURE}

In 1945, before the advent of ultrasound, the possibility was mentioned of a multiple gestation conception rate that was higher than previously had been believed. Stoeckel said, "It thus appears that twins are more often conceived than born; not only in addition to the evidence of foeti papyracei, it may be that twin material is reabsorbed due to early death, without leaving any trace" [38]. Moreover, Stoeckel quoted Verschuer as having postulated a $68 \%$ "disappearance" rate of multiple conceptions [38]. Following the widespread utilization of ultrasound in recent years, nine papers have documented the phenomenon of the "vanishing twin" (Table 1).

Hellman et al [10] in 1973 studied 140 women with histories of previous obstetric difficulties. From a total of 22 patients with multiple gestational sacs demonstrable on ultrasound examination during the first trimester (nine of whom received artificial ovu- 
lants), only five sets of twins and three singletons were delivered. The remaining 14 women aborted. The authors postulated that the double gestational sacs appearing spontaneously in the 13 women who had not received artificial ovulants represented monovular twinning. Thus, the frequency of monovular twinning was approximately 25 times higher than the expected rate, or nearly $10 \%$.

In 1974, Kohorn and Kaufman [15] described three patients in whom double gestational sacs were seen in a prospective study of 65 women scanned by ultrasound during the first trimester of pregnancy. All three women delivered singletons; the authors noted, however, that the double sacs were seen only at a single observation, and this finding could not be repeated on subsequent scans.

Levi's classic report [20] in 1976 describes a cohort of 6,990 women, all of whom received ten separate ultrasound scans. A total of 118 patients were shown to have multiple sacs. Of 28 women scanned prior to the tenth week of gestation, follow-up data were available for 14 . Of these 14 patients, only four sets of twins $(28.6 \%)$ were delivered. Among 11 women diagnosed as having multiple gestations between the tenth and the 15 th weeks, eight were followed through delivery; only three sets of twins $(37.5 \%)$ were delivered. Multiple gestation was diagnosed in 79 patients scanned after the 15 th week. Here, 77 sets of twins and one set of triplets resulted; in one woman, a fetus papyraceus was diagnosed by scan during the 17 th week, but the coexisting singleton developed normally. Levi thus reported a $71 \%$ "disappearance" rate of twin gestation when diagnosis was made prior to the tenth week.

In 1977, Robinson and Caines [35] reported on 30 women with multiple gestational sacs demonstrated by ultrasound during the first trimester. In 14 cases where twins were delivered, five mothers had received artificial ovulants. Ten women whose scans confirmed the presence of a normal pregnancy and a coexisting blighted ovum delivered singletons; one patient with the same ultrasonic diagnosis aborted at 25 weeks after ovulation induction. The remaining five women in the study aborted. Sonar examination of these five women demonstrated four cases each with blighted ovum/blighted ovum and one case with blighted ovum/missed abortion. The only antepartum findings consisted of (1) a macerated fetus seen in the products of an incomplete abortion in one patient with sonar evidence of blighted ovum/missed abortion and (2) a flattened, empty sac on the fetal side of the placenta in the patient whose scans indicated the presence of a normal pregnancy with coexisting blighted ovum. This study suggests that the true incidence of multiple pregnancy is at least one in 60 .

Levi and Reimers [22] studied the phenomenon of the "vanishing twin" in 1978. They documented 159 patients with multiple gestational sacs among 3,161 women who were scanned by ultrasound during the first trimester. Forty-seven women were diagnosed with multiple gestational sacs between the fourth and ninth weeks ("certain" diagnosis in 34, "probable" in 8 , and "doubtful" in 5). Of these 47 pregnancies, follow-up was available for 32 , only six of which resulted in a multiple birth. Of 23 patients diagnosed with multiple pregnancy between ten and 14 weeks of gestation ("certain" diagnosis in ten, "probable" in six, and "doubtful" in seven), 22 were followed through delivery. Only 14 of these 22 women delivered multiples. All of the 89 women diagnosed with multiple gestation after 14 weeks delivered multiples; in all of them the diagnosis was "certain." This study cites a twin "disappearance" rate of $78 \%$, if a "certain" diagnosis is made by scan prior to the tenth week of gestation. The authors calculated a $1.9 \%$ incidence of multiple pregnancy on the basis of their observation of 159 multiple sacs in their total patient population of 8,362 women. 


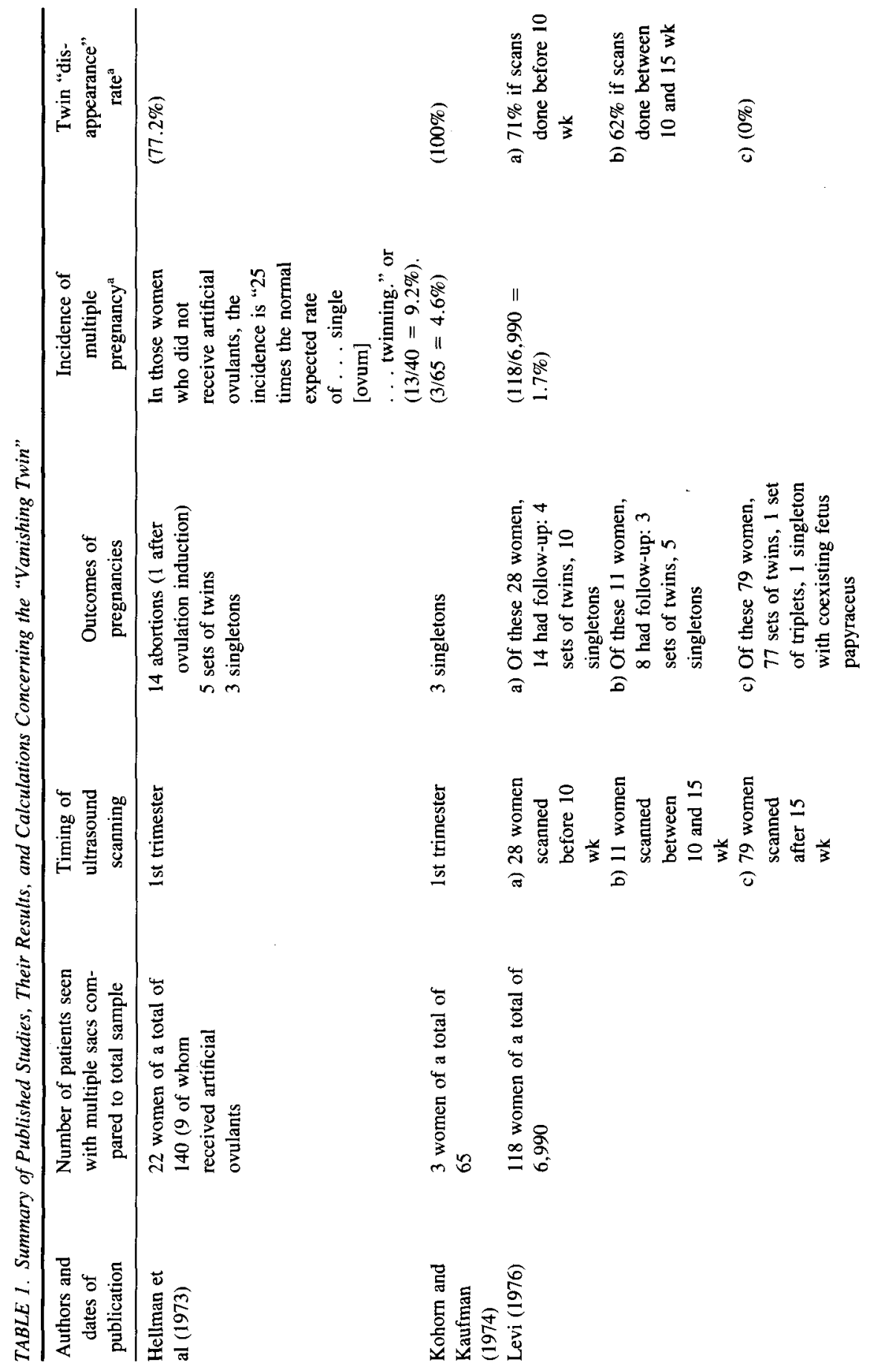




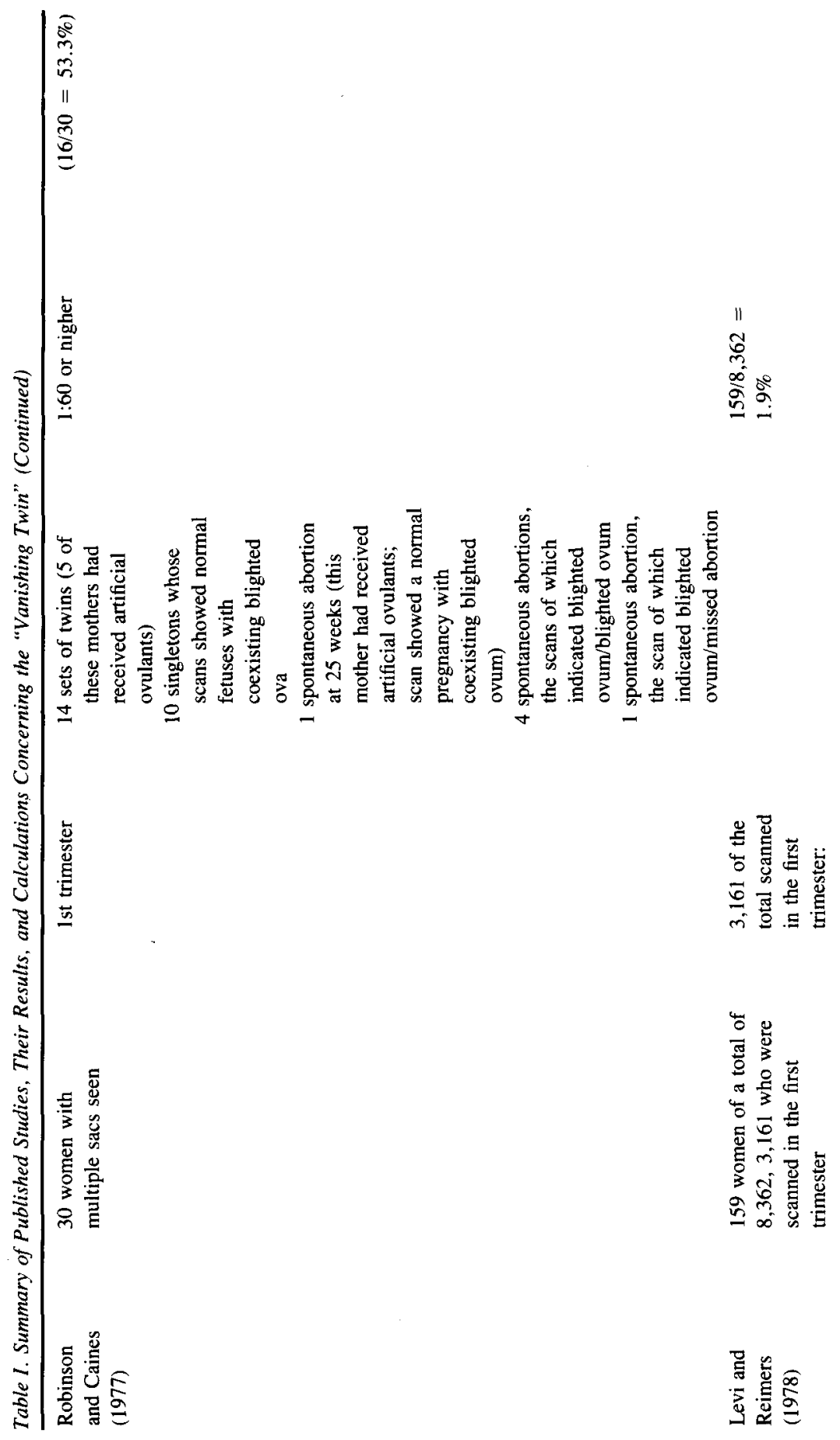




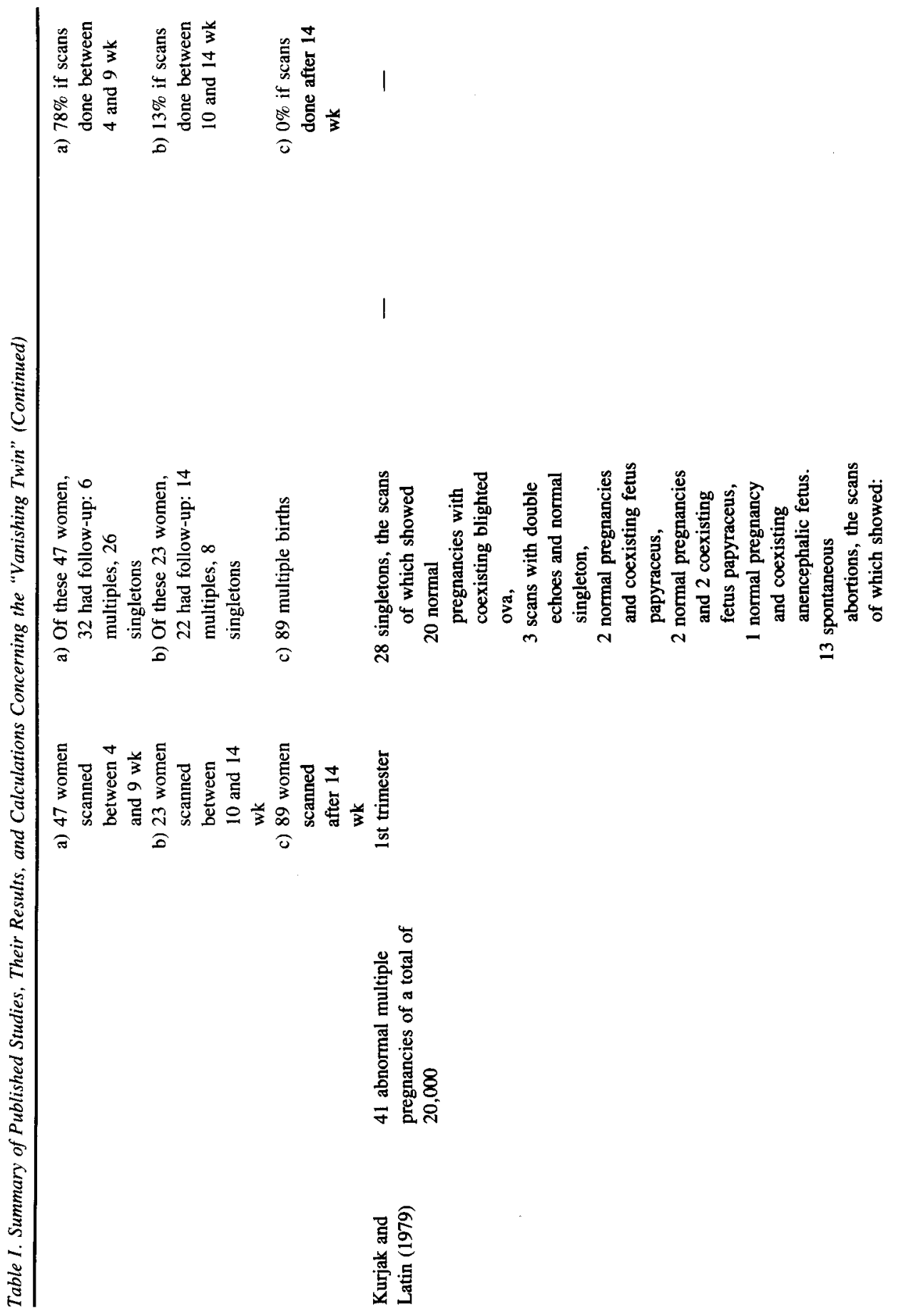




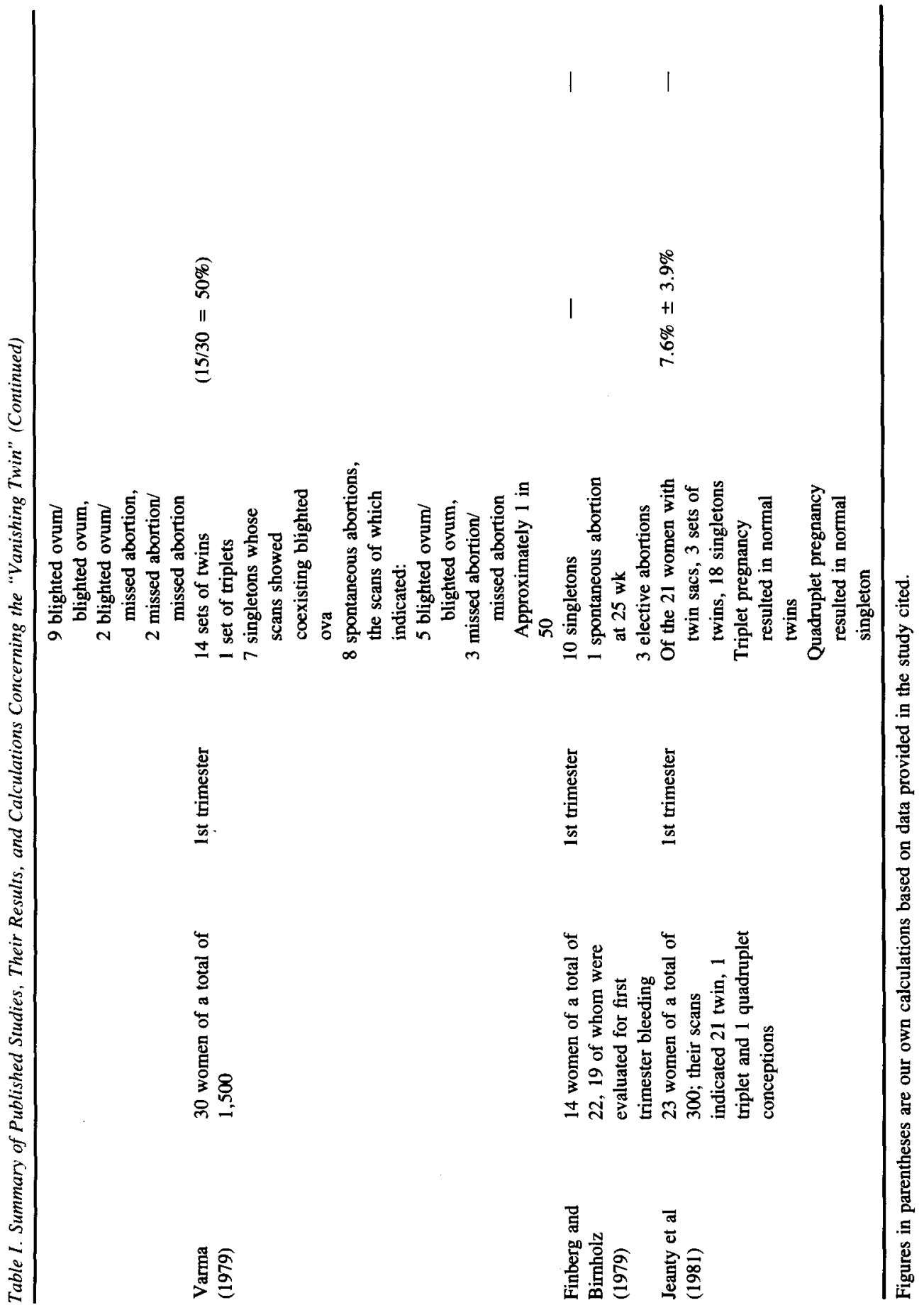


Kurjak and Latin [17] in 1979 described 41 cases of abnormal multiple pregnancies detected over a four-year period from a cohort of 20,000 women scanned with ultrasound during the first trimester. Of these, only eight singletons were delivered. However, other findings include 20 patients with normal singleton pregnancies coexisting with blighted ova; nine patients each with blighted ovum/blighted ovum; three women whose scans showed double echoes but in whom only one fetus ultimately developed and was delivered; two patients with blighted ova and coexisting missed abortions; one normal singleton pregnancy with coexisting anencephalic fetus; two cases of a singleton pregnancy coexisting with a fetus papyraceus; and two women with normal singleton pregnancies coexisting with two fetuses papyraceus. Postpartum evidence of the "disappearing" twin or triplet was sought in each of the singleton deliveries, but none was found.

Varma [41] in 1979 studied 1,500 women with ultrasound during the first trimester. He found 30 patients with multiple gestational sacs and calculated an incidence of multiple pregnancy of one in 50 . Of the 30 patients, 15 sets of multiples were delivered (14 sets of twins and one set of triplets). The remaining pregnancies resulted in seven singleton deliveries (each having been associated with a coexisting blighted ovum) and eight spontaneous abortions (three patients each with missed abortion/missed abortion and five patients each with blighted ovum/blighted ovum). Additionally, for 12 women with complications of first trimester vaginal bleeding, 11 pregnancies terminated in spontaneous abortion (36.7\%).

A 1979 paper by two radiologists, Finberg and Birnholz [8], described the ultrasonograms of 22 women scanned during the first trimester, 19 of whom presented with first trimester bleeding. Fourteen patients were found to have double sacs. Of these, ten singletons were delivered, three pregnancies were terminated by elective abortions, and one pregnancy terminated in spontaneous abortion at 22 weeks. Interestingly, in addition to a normal fetus, the authors identified an abnormal intrauterine fluid locule on the scans of all of the original patients in the study. This abnormal zone was postulated to represent a "blighted twin." The authors noted no postpartum evidence of this abnormal area in any of the singleton deliveries. In one of the elective abortions, however, they identified an area containing $30 \mathrm{ml}$ of dark brown altered blood that was consistent with an ultrasonic interpretation of a hemorrhagic second sac.

A recent paper by Jeanty et al [13] discusses a prospective study of 300 pregnancies, all of which were scanned by ultrasound. Two hundred twenty-five women were scanned prior to the ninth week, and the remaining 75 before the 14th week. From the entire group, 23 multiple pregnancies were identified: 21 twin, one triplet, and one quadruplet. Six of these 23 patients were referred for clarification or documentation of the "haemorrhage or miscarriage syndrome." Only three of the 21 women believed to be carrying twin pregnancies ultimately delivered twins, however. The authors stressed that "only six pregnancies were seen for the first time as normal twin gestations." The triplet pregnancy resulted in the delivery of normal twins, and the quadruplet pregnancy resulted in the delivery of a normal singleton. These authors described three different ultrasonic aspects of the "vanishing twin" which could be identified before the complete "disappearance" of a gestational sac (see Discussion). They also calculated a very high rate of multiple pregnancy $(7.6 \% \pm 3.9 \%)$.

\section{MAIL SURVEY}

One hundred fifty-five letters were sent to members of the International Society for Twin Studies. Several recipients forwarded their letters to persons more familiar with the 
"vanishing twin" concept. In all, there were 28 replies. Some respondents indicated that the concept of the "vanishing twin" was not unknown to them; few had dealt directly with instances of the phenomenon among their own patients. Thiery and Defoort $[39,40]$ stated they never had seen a "twin actually vanishing" among the approximately 2,800 ultrasound examinations perforrned annually at the Akademisch Ziekenhuis in Belgium. They had, however, evaluated a few cases of early second trimester bleeding "where a second amniotic sac (empty) seemed to exist in the lower uterine segment" [40]. They postulated that this apparently empty sac might be an area involving dissolution of the decidua and thus the cause of the bleeding as opposed to being a "vanishing twin."

Benirschke [2] from the Zoological Society of San Diego in California readily acknowledged that the "diagnosis of vanishing twin has . . . been made a number of times." In the three or four cases reported to him, however, he was unable to find postpartum evidence of a remnant of a twin or a second sac after careful examination of the placenta and membranes. Benirschke reported a case of a 37-year-old woman with a twinning history whose pregnancy resulted in "one liveborn normal twin, [and] a diminutive second (dichorionic) placenta with a two-centimeter . . . macerated fetus," which he estimated to have died at approximately 8 weeks of age.

MacGillivray [25] from the University of Aberdeen in Scotland replied with fascinating personal letters from numerous women relating histories of having successful deliveries of singleton infants after having had the diagnosis of spontaneous abortion confirmed by their obstetricians earlier in the same pregnancy. Many of these reports were from women in whom this phenomenon had occurred some 20 to 50 years earlier, long before any ultrasound confirmation was possible.

Our own survey of obstetricians affiliated with Northwestern University Medical School in Chicago, Illinois, resulted in five replies. These physicians recalled approximately eight cases where "vanishing twins" occurred on the basis of ultrasonic examinations performed under the supervision of Rudy E. Sabbagha, MD, Chief of the Division of Ultrasonography, at the Prentice Women's Hospital and Maternity Center in Chicago, Illinois.

\section{DISCUSSION}

Serial ultrasonograms on 172 women described in the studies above document the "disappearance" of one or more gestational sacs. The rate of "disappearance" varies considerably; because of differences in study methodology, it is not practical to calculate an average rate. Several explanations have been proposed to account for the phenomenon of the "vanishing twin." In the following sections, various theories accepting as well as refuting a high incidence of multiple conception will be discussed. In addition, sources of error in the overdiagnosis and underdiagnosis of multiple pregnancy by ultrasound will be enumerated.

\section{Physiological Explanations}

Resorption. Resorption is the most apparent explanation for the "disappearance" of a gestational sac. Resorption occurs in singleton pregnancies and in lower animals [7,42]. Some investigators consider this phenomenon a plausible explanation for the "disappearance" of one or more gestational sacs in a human multiple pregnancy $[10,13,18,20]$. Although resorption of a gestational sac can occur early in pregnancy, the timing is variable. Levi [20] reported resorption by the seventh or eighth week of gestation, Ro- 
binson [7] observed the phenomenon by the ninth week, and Reinold [30] opined that a gestational sac may be resorbed as late as the 12th week. Although resorption is probably responsible for the "disappearance" of a certain proportion of gestational sacs, the true incidence of resorption is unknown. It is clear, however, that resorption of one or more gestational sacs during pregnancy apparently takes place without adverse effect on any coexisting fetus or fetuses $[13,16]$.

Blighted Ovum. A second physiological explanation for the "disappearance" of a viable gestational sac is the blighted ovum or anembryonic pregnancy. According to Robinson $[32,35]$, numerous gestational sacs which "disappeared" on serial ultrasound examinations were subsequently found to be blighted ova. By definition, these are gestational sacs of at least $2.5 \mathrm{ml}$ in volume in which no fetus can be visualized on ultrasound examination [35]. Within blighted ova, however, small echoes have occasionally been identified $[8,13]$.

The scans of 22 women in the Finberg and Birnholz study [8] demonstrated a separate anechoic or hypoechoic area within the uterus either in close proximity or adjacent to the normal amniotic sac. These authors suggested that this finding was compatible with an anembryonic pregnancy or "blighted twin" coexisting with a normal pregnancy. Three different ultrasonographic patterns were identified: (1) a second sac, either empty or with some internal echoes (found in 14 of the 22 women); (2) a septal division of the amniotic cavity with one compartment empty; and (3) a fluid crescent outlining the intact gestational sac. Finberg and Birnholz suggested that the first two patterns represented different stages of the same "blighting" phenomenon, in which the second pattern evolved as the two sacs enlarged and came into contact, eventually forming a common boundary between them. The pathogenesis of the third pattern was uncertain, however.

Jeanty et al [13] described three ultrasonic observations associated with the "vanishing" phenomenon prior to complete "disappearance": (1) a smaller than normal configuration of the gestational sac with irregular margins, poor turgescence, and an incomplete trophoblastic ring; (2) a crescent-shaped gestational sac with incomplete trophoblastic ring, "in which the gestational sac shares an important part of the interamniotic membrane"; and (3) a small echogenic spot corresponding to a shrunken gestational sac. These three patterns were followed using serial ultrasonic examinations. The authors found that the "vanishing twin" may evolve from the "smaller than normal configuration" into either pattern 2 or pattern 3. However, the second pattern, that of the "crescent shape," was not shown to develop into the "small echogenic spot." The authors postulated that

... if the vanishing process takes place at a very early stage, when the gestational sac is filled with a very small amount of fluid, . . . evolution to an echogenic spot may take place. If the vanishing process takes place later on, [however,] the resorption of the fluid seems to occur against an active or passive mechanism of liquid production and the crescent shape may grow and finally regress or persist. [13]

Whether these patterns actually describe the events prior to the formation of a blighted ovum was not discussed.

Several studies propose that the only apparent complication of regression of a blighted ovum is slight vaginal bleeding $[8,17,35]$. Varma [41] stated that his patients with multiple conceptions had a relatively high incidence of first trimester bleeding $(40 \%)$, whereas those women studied by Jeanty et al [13] had a relatively low incidence of this problem (six patients of 23 , or $26.1 \%$ ). On the other hand, Thiery and Defoort $[39,40]$ are skeptical as to whether first trimester bleeding associated with an anembryonic sac on ultrason- 
ography always represents a blighted ovum. They hypothesize that the area in question, occurring in the lower uterine segment in the cases they studied, may merely represent an area of dissolution of the decidua and that this process may originally have caused the vaginal bleeding.

Finberg and Birnholz [8] found a high percentage of multiple gestational sacs in the women referred to them for first trimester bleeding. Regardless of the vaginal bleeding that may be associated with regression of a blighted ovum, a normal pregnancy which coexists alongside a blighted ovum has a good prognosis for carrying to term $[8,13,35]$. For this reason, Finberg and Birnholz have advised that all women bleeding in the first trimester be examined by ultrasound before undergoing a therapeutic dilation and curettage to prevent the inadvertent abortion of the remaining twin.

Fetus Papyraceus. The simultaneous occurrence of a fetus papyraceus in the presence of a viable twin is rare; the incidence is reported to be 1:12,000 live births and 1:184 twin births [29]. No consensus exists regarding the effect of a fetus papyraceus on the mother or on the coexisting viable fetus. In the series reported by Livnat et al [29], hypertension was noted in two of the three women. In only one of the pregnancies, however, did the mother deliver a liveborn singleton in addition to the fetus papyraceus. The authors concluded that the appearance of a fetus papyraceus may frequently indicate the presence of a hostile intrauterine environment. In discussing the blighting phenomenon, Livnat et al have suggested that

a "disappearing" twin on serial ultrasonic examinations is not necessarily due to fetal movements, diagnostic error, or complete reabsorption, and the possibility of a fetus papyraceus must be kept in mind. [29]

\section{Arguments Critical of the High Rate of Multiple Gestation}

Although numerous physicians accept the existence of the "vanishing twin" phenomenon, many are skeptical as to whether it occurs as frequently as can be inferred from some of the data presented above. In support of this statement, pathological evidence to confirm "disappearance" rates as high as $\mathbf{7 8 \%}$ is lacking. Indeed, examination of the placenta and membranes after the birth of a singleton originally described by ultrasound as being one of a pair of twins rarely shows evidence of the supposedly "vanished" twin. Similarly, examination of the products of abortion in search of evidence of "disappearing" gestational sacs generally is unproductive.

Of the publications cited in this review, only two offer concrete pathological evidence of the "vanished" twin. Robinson and Caines [35] reported one example among their 30 patients: the fetal surface of the placenta demonstrated a flattened and empty sac, $3 \mathrm{~cm}$ in diameter. Finberg and Birnholz [8] identified an area containing $30 \mathrm{ml}$ of dark brown altered blood in the pathological specimen of an elective abortion; this observation was consistent with the appearance of a hemorrhagic second sac seen on ultrasound examination. No other studies, either published or made available to us through personal correspondence, have revealed any confirmatory postpartum or pathological evidence of the "disappearing" twin gestation.

The lack of such pathological evidence is not surprising in view of the inherently small area in question. According to Finberg and Birnholz:

If the pregnancy terminates in abortion, the products of conception are generally disrupted and fragmentary. If the pregnancy continues to term, the second sac may have been expelled, 
fragmented or resorbed, or may persist as a small atretic region overlooked even after careful scrutiny of the placenta and membranes. [8]

Similar comments have been made by Robinson and Caines [35].

\section{Sources of False-Positive Errors in the Overdiagnosis of Multiple Gestations: Attempts to Explain the High Rate of Twin "Disappearance"}

In attempting to explain the high twin "disappearance" rate reported in the studies reviewed here, several potential errors in technique and interpretation merit discussion. One of the most obvious sources of false-positive errors involves the use of pictures produced by low-quality ultrasound equipment which lack the resolution capacity of today's machines [40]. A second potential source of error is artifact. Simple technical artifacts are known to distort the echographic images of normal singleton pregnancies. If viewed early in a woman's pregnancy and not recognized as such, an artifact theoretically could result in false interpretations that later could affect the care of the pregnancy itself [22]. Several authors note that artifacts can be minimized as a source of error by either increasing or decreasing the power level of the ultrasound equipment in an attempt to adjust the sonographic picture. In that way, an image not due to artifact would not disappear with a small change in the energy output $[29,35]$.

Single ultrasonic scans may not permit optimal viewing of the uterine cavity; they may also incorrectly suggest the presence of defects or septa of the gestational sac $[13,15]$. For example, a single hourglass-shaped gestational sac may incorrectly be interpreted as more than one sac and as being indicative of the presence of a multiple conception $[5,30]$. Moreover, fetal echoes which reflect fetal movement may incorrectly convey the impression of more than one gestational sac, especially since the fetus usually is quite active by the eighth week of pregnancy $[4,5,36]$. Jeanty et al [13] also have noted that small echoes may be present within a blighted ovum, and occasionally these may be misinterpreted as indicating a viable fetus.

Overdiagnosis of multiple gestation also may occur because of incorrect interpretation of the physiological cavities which normally are present early in fetal development. Examples of this phenomenon include the following: (1) The amniotic cavity is a small bubble within the chorionic sac until the tenth or twelfth week of gestation; after this date the amnion applies itself to the inside of the chorion $[2,30]$. (2) The extraembryonic celom encloses the amniotic cavity early in pregnancy and eventually disappears [30]. (3) The yolk sac can be seen as a 2-5-ml spheric structure lying adjacent to the fetus by approximately the seventh to 11 th weeks [26]. (4) The uterine cavity is not visualized ultrasonically as a well-demarcated structure [30].

Jeanty et al [13] were able to identify physiological conditions that mimicked two of the three ultrasonic patterns they described. These authors noted that the "smaller than normal gestational sac" pattern was perfectly imitated on ultrasonography by a decidual reaction in the second horn of a bicornuate uterus. Furthermore, the "crescent shape" pattern was found to be simulated by an intrauterine hematoma after extraction of an intrauterine device.

\section{Sources of False-Negative Errors in the Underdlagnosis of Multiple Conceptions}

A major source of the underdiagnosis of multiple pregnancy is failure to carefully and completely scan the lower uterine segment. After a single gestational sac or fetal head 
is identified, the tendency is to terminate an ultrasound examination [30]. Such an action precludes the identification of one or more additional fetuses.

A second source of error is related to the transient nature of multiple sacs during the first and early second trimesters [34,35,41]. From longitudinal studies of early pregnancies, Robinson $[34,35]$ has concluded that a second sac might be visible for only a period of one to two weeks and that, once it appeared, it did not reappear on subsequent ultrasound examinations. In a personal communication, Robinson has indicated that

... these abnormal "blighted" sacs grow more slowly, and are therefore often not visible as early as a normally developing sac, and that with the growth of the normal sac, they will be compressed against the uterine wall. [34]

Varma [41] also believes that a substantial number of multiple gestational sacs may be missed because of the transient nature of these abnormal sacs and notes that the patient may not be referred for ultrasound examinations so early in pregnancy.

\section{The "Vanishing Twin": Interrelatlonship With First Trimester Bleeding and Spontaneous Abortion}

This issue has been addressed by several investigators. Any pregnancy complicated by first trimester bleeding is known to have an increased risk of spontaneous abortion, although the presence of bleeding does not always lead to a spontaneous abortion. Reports are in agreement that first trimester bleeding is the only complication thus far known that occasionally occurs with the "disappearance" of a fetus $[8,17,35,41]$. Several authors have stressed that "disappearance" of a gestational sac need not be associated with impending spontaneous abortion, however $[15,17]$.

Although Varma's study [41] indicates an unusually high incidence of spontaneous abortion (36.7\%), most other investigations do not confirm this finding. Finberg and Birnholz [8] reported that only one of 14 women aborted (7.1\%), and Robinson and Caines [35] stated that only six spontaneous abortions occurred among the 30 women they studied (20\%). Higher spontaneous abortion rates were shown in the papers by Kurjak and Latin [17], who reported 13 of 41 patients who spontaneously aborted $(31.7 \%)$, and by Hellman et al [10], who documented 14 spontaneous abortions among 22 women $(63.6 \%)$. It must be recalled, however, that the pregnancies identified in the study by Kurjak and Latin [17] were described as "abnormal multiple pregnancies" and that Hellman et al [10] reported on a select group of women known to have previous obstetric difficulties. None of the other studies included any multiple pregnancy that resulted in a spontaneous abortion.

Except for the high incidence of spontaneous abortion that may be associated with the formation of a fetus papyraceus [16], the incidence of spontaneous abortion in the patients studied in the papers reviewed here is relatively low. When methods of "disappearance" of a gestational sac in utero are discussed, the fetus papyraceus must be considered separately, for its presence alone suggests a hostile intrauterine environment [16]. Therefore, if the formation of a fetus papyraceus is not considered, the studies reviewed here indicate that after "disappearance" of a gestational sac on ultrasound examination, the prognosis for carrying the coexisting viable twin to term is good $[7,8,35]$.

From these studies, we assessed the risk of spontaneous abortion when a gestational sac had "disappeared" on serial ultrasound examinations and the pregnancy was also complicated by first trimester bleeding. Only two of the nine studies reviewed here 
included information regarding first trimester bleeding and outcome of pregnancy. The risk of spontaneous abortion in a "vanishing twin" pregnancy complicated by first trimester bleeding ranges from $26.3 \%$ (five of 19 patients in the study by Finberg and Birnholz [8]) to $91.6 \%$ (11 of 12 patients in Varma's study [41]).

\section{The "Vanishing Twin" and the Use of Artificial Ovulants}

It is interesting to consider the outcomes of those pregnancies with multiple sacs resulting from the use of artificial ovulants to enhance fertility. The use of artificial ovulants is known to increase the incidence of multiple births; the incidence of multiple gestation ranges from $11 \%$ to $44 \%$ after treatment with gonadotropins [9] and from $6 \%$ to $12 \%$ after treatment with clomiphene [1]. The incidence of spontaneous abortion associated with the use of artificial ovulants has been reported to range from $10 \%$ to $13 \%$ after the use of gonadotropins [9] and from $11 \%$ to $30 \%$ after the use of clomiphene [1].

Only two investigators included fertility information in their reports on the "vanishing twin" phenomenon. Nine of the 22 women found to have multiple gestational sacs in the study by Hellman et al [10] had received artificial ovulants. Of these nine, there were five sets of twins, three singletons, and one spontaneous abortion; the remaining 13 women with spontaneously appearing double sacs all aborted. In the study by Robinson and Caines [35], at least six women received artificial ovulants. Five of these patients delivered twins, and one patient with the ultrasonic diagnosis of a normal pregnancy coexisting with a blighted ovum spontaneously aborted at 25 weeks. The chances of carrying to term a pregnancy conceived with the use of fertility agents is therefore quite good, with only two spontaneous abortions occurring in a total of 15 women treated with artificial ovulants.

\section{CONCLUSIONS}

On the basis of our literature search and mail surveys, we conclude that the phenomenon of the "vanishing twin" does exist.

1. The incidence of human multiple conception is higher than has previously been believed. Accurate assessment of the incidence of "vanishing twins," however, is difficult, mainly because it is not routine for pregnant women to undergo early first trimester ultrasound examinations. Available series of first trimester and early second trimester ultrasound scans cite varying frequencies of twin "disappearance," ranging from $0 \%$ to $78 \%$, depending on the patient population studied, the timing of ultrasonography and the number of ultrasound scans performed (Table 1).

2. In general, higher "disappearance" rates have been noted in patients whose scans were performed earlier (before the tenth week) than later in pregnancy (later than the tenth to 14th week of gestation). These higher values reflect several confounding phenomena. It is difficult to accurately diagnose multiple pregnancies by identifying more than one gestational sac by ultrasonography prior to the 12th week of gestation. Falsepositive errors in the overdiagnosis of multiple conceptions may occur. As the pregnancy continues, the accuracy of correctly diagnosing the presence of more than one fetus by ultrasonography increases.

3. Although the exact mechanisms of "disappearance" are unclear at present, the "vanishing" phenomenon occurs during the latter half of the first trimester or the early part of the second trimester of pregnancy. Physiological explanations for the "disappearance" of an embryo or fetus in utero include resorption and formation of blighted 
ova or fetuses papyraceus. The true numbers of "vanishing twins" that "disappear" through any of these physiological conditions are not known.

4. Thus far, the only apparent complication associated with "disappearance" of a fetus is slight vaginal bleeding. The prognosis for carrying the coexisting viable twin to term, however, is good despite the occurrence of vaginal bleeding. The data strongly suggest that all women with first trimester bleeding should be examined by ultrasound before undergoing a therapeutic dilation and curettage, in order to prevent inadvertent abortion of a remaining fetus.

5. The use of early ultrasonic examinations can identify the presence of multiple gestational sacs. An obstetrician should carefully consider the variable rates of "disappearance" of gestational sacs in the studies reviewed here before informing the mother of a possible diagnosis of multiple pregnancy. Because of the social and emotional impact, as well as the physical and financial burdens added by the presence of any set of multiple children, an accurate, faultless diagnosis is demanded of the obstetrician.

\section{ACKNOWLEDGMENTS}

We wish to extend special thanks to Catherine Brewer, Renee Jacquin, Ruth Perelmuter, and Drs Rudy Sabbagha and Charles E. Boklage.

\section{REFERENCES}

1. Asch RH, Greenblatt RB (1976): Update on the safety and efficacy of comiphene citrate as a therapeutic agent. J Reprod Med 17(3):175-180.

2. Benirschke K (July, 1981): Personal communication.

3. Benirschke K, Driscoll SG (1967): "The Pathology of the Human Placenta." New York: Springer-Verlag, p 162.

4. Cadkin AV, Motew MN (1979): "Clinical Atlas of Gray Scale Ultrasonography in Obstetrics." Springfield, Illinois: Charles C. Thomas, pp 269, 272.

5. Defoort P, Van Eyck J, De Schryver D, Thiery M (1976): Early diagnosis of twin pregnancy by ultrasonography: Sources of false positive errors. IRCS Medical Science: Biomedical Technology; Clinical Medicine; Reproduction, Obstetrics and Gynecology 4:166-167.

6. Donald I (1967): Sonar in obstetrics and gynecology. In "Year Book of Obstetrics and Gynecology," JP Greenhill (ed): Chicago: Year Book, p 242.

7. Dziuk P (March, 1982): Personal communication.

8. Finberg HJ, Birnholz JC (1979): Ultrasound observations in multiple gestation with first trimester bleeding: The blighted twin. Radiology 132:137-142.

9. Hack M, Lunenfeld B (1979): The influence of hormonal induction of ovulation on the fetus and newborn. Pediatr Adolesc Endocrinol 5:191-212.

10. Hellman LM, Kobayashi M, Cromb E (1973): Ultrasonic diagnosis of embryonic malformations. Am J Obstet Gynecol 115(5):615-623.

11. Hewitt D, Stewart A (1970): Relevance of twin data to intrauterine selection: Special case of childhood cancer. Acta Genet Med Gemellol 19:83-86.

12. Javert CT (1957): "Spontaneous and Habitual Abortion." New York: Blakiston Division, McGraw-Hill Book Co., Inc., p 206.

13. Jeanty P, Rodesch F, Verhoogen C, Struyven J (1981): The vanishing twin. Ultrasonics 2:25-31.

14. Keith L, Hughey MJ (1979): Twin gestation. In Sciarra JJ (ed): "Gynecology and Obstetrics, Vol. 2." Hagerstown, Maryland: Harper and Row, p 1.

15. Kohom EI, Kaufman M (1974): Sonar in the first trimester of pregnancy. Obstet Gynecol 44(4):473-483.

16. Kurjak A (1980): Fetal abnormalities in early and late pregnancy. In: "Progress in Medical Ultrasound: Reviews and Comments, Vol. 1." Amsterdam-Oxford: Exerpta Medica, pp 117-119.

17. Kurjak A, Latin V (1979): Ultrasound diagnosis of fetal abnormalities in multiple pregnancy. Acta Obstet Gynecol Scand 58:153-161. 
18. Levi S (1978): Abnormalities in early pregnancy. In de Vlieger (ed): "Handbook of Clinical Ultrasound." New York: John Wiley and Sons, pp 138-139.

19. Levi S (1976): Diagnostic ultrasound in early pregnancy. In Donald I, Levi S (eds): "Present and Future of Diagnostic Ultrasound." New York: John Wiley and Sons, pp 92-93.

20. Levi S (1976): Ultrasonic assessment of the high rate of human multiple pregnancy in the first trimester. J Clin Ultrasound 4(1):3-5.

21. Levi S, DeBock G (1972): Le diagnostic précoce des grossesses multiples par les ultra-sons. J Radiol Electrol 53:690-693.

22. Levi S, Reimers M (1978): Démonstration échographique de la fréquence relativement élevée des grossesses multiple humaines pendant la période embryonnaire. In: "L'Implantation de L'Oeuf." Paris: Masson, pp 295-307.

23. Livingston JE, Poland BJ (1980): A study of spontaneously aborted twins. Teratology 21:139-148.

24. Livnat EJ, Burd L, Cadkin A, Keh P, Ward AB (1978): Fetus papyraceus in twin pregnancy. Obstet Gynecol 51(1)(suppl):41s-45s.

25. MacGillivray I (July, August, 1981): Personal communication.

26. Mantoni M, Pedersen JF (1979): Ultrasound visualization of the human yolk sac. J Clin Ultrasound 7:459-460.

27. Miller JF, Williamson E, Glue J, Gordon YB, Grudzinskas JG, Sykes A (1980): Fetal loss after implantation: A prospective study. Lancet 2:554-556.

28. Milne B (1980): Problems in ultrasonic monitoring of multiple pregnancies. Br J Radiol 53:85-86.

29. Ottolenghi-Preti GF (1972): Sopra un rarissimo caso di gravidenza gemellare con un feto papiraceo e con inserzione velamentosa del funicolo del feto vivo. Ann Oste Finecol Med Perinat N3 XCIII:173. (Quoted in Livnat et al, reference \#24).

30. Reinold E (1976): "Ultrasonics in Early Pregnancy." New York: L. Karger-Basel, pp 96-98.

31. Roberts J, Lowe CR (1975): Where have all the conceptions gone? Lancet 1:498-499.

32. Robinson HP (1975): The diagnosis of early pregnancy failure by sonar. Br J Obstet Gynaecol 82(11):849-857.

33. Robinson HP (1978): Normal development in early pregnancy. In de Vlieger (ed): "Handbook of Clinical Ultrasound." New York: John Wiley and Sons, p 122.

34. Robinson HP (December, 1981): Personal communication.

35. Robinson HP, Caines JS (1977): Sonar evidence of early pregnancy failure in patients with twin conceptions. Br J Obstet Gynaecol 84:22-25.

36. Sabbagha RE (December, 1981) Personal communication.

37. Sabbagha RE (1979): "Ultrasound in High-Risk Obstetrics." Philadelphia: Lea and Febinger, p 32.

38. Stoeckl W (1945): "Lehrbuch der Geburtschilfe." Jena: Gustav Fischer. (Quoted in Levi, reference \#20).

39. Thiery $M$ (November, 1981): Personal communication.

40. Thiery M, Defoort P (June, 1981): Personal communication.

41. Varma TR (1979): Ultrasound evidence of early pregnancy failure in patients with multiple conceptions. Br J Obstet Gynecol 86:290-292.

42. Webel SK, Dziuk PJ (1974): Effect of stage of gestation and uterine space on prenatal survival in the pig. J Animal Sci 38(5):960-963.

Correspondence: Louis Keith, MD, 333 East Superior Street, Suite 463-5, Chicago, Illinois 60611. 